\title{
Understanding the Cap Structure in K2P Channels
}

\author{
Leandro Zúñiga * and Rafael Zúñiga \\ Escuela de Medicina, Centro de Investigaciones Médicas, Universidad de Talca, Talca, Chile
}

Keywords: K2P channels, two-pore domain channel, cap structure, K2P potassium channels structure, potassium channels

The two-pore domain potassium (K2P) channel family is composed by 15 members, identified in the human genome, and also K2P channels have been identified in yeast, plants, zebrafish, nematode and fruitfly (Goldstein et al., 2001). Based on their primary structure and functional properties, K2P channels are grouped into six distinct subfamilies denoted as TREK, TALK, TASK, TWIK, THIK, and TRESK (Goldstein et al., 2001, 2005; Figure 1A).

$\mathrm{K} 2 \mathrm{P}$ channels play several key roles in excitable cells. For instance, K2P channels generate the called leak or background currents that are responsible for the maintenance of the resting membrane potential close to the equilibrium potential of $\mathrm{K}^{+}\left(\mathrm{E}_{\mathrm{K}}\right)$ which sets the resting membrane potential below the firing threshold (Lesage and Lazdunski, 2000; Goldstein et al., 2001; Lotshaw, 2007; Enyedi and Czirjak, 2010).

K2P channels have also been linked to several pathologies. For example, TASK-1 malfunction as consequence of missense mutations or its pharmacological inhibition has been associated to pulmonary hypertension (Ma et al., 2013) and cardiac arrhythmias (atrial fibrillation) (Schmidt et al., 2015), respectively. Additionally, mutations of TASK-3 (G236R) and TRESK are linked with the Birk Barel syndrome (Barel et al., 2008) and familial migraine (Lafreniere et al., 2010). On the other hand, an overexpression of TASK-3 has been found in some human breast cancer tumors where it has been proposed acts as a proto-oncogene (Mu et al., 2003). For comprehensive reviews see Enyedi and Czirjak (2010) and Feliciangeli et al. (2015). Taken together, K2P channels could be potential pharmacological targets for restoring the function of dysfunctional excitable tissues as well as in cancer treatment.

The open probability of $\mathrm{K} 2 \mathrm{P}$ channels is highly regulated by a wide variety of stimuli such as kinases, phosphatases, lipids, G proteins, internal and external $\mathrm{pH}$, mechanical force, protein-protein interactions and volatile anesthetics (Lesage and Lazdunski, 2000; Goldstein et al., 2001; Lotshaw, 2007; Enyedi and Czirjak, 2010).

$\mathrm{K} 2 \mathrm{P}$ channels display a structure that markedly differs to that described for $\mathrm{Kv}$ and $\mathrm{Kir} \mathrm{K}^{+}$ channel families. Each K2P subunit consists of four transmembrane domains (M1-M4 domains), two pore forming domains (P domains) with both $\mathrm{N}$ - and $\mathrm{C}$ - termini facing the cytosolic side (Figure 1B). Given that $4 \mathrm{P}$ domains are required to form a $\mathrm{K}^{+}$selectivity filter, it was assumed that the K2P channels must function as dimers. Strong evidence to support this architecture was initially reported (Lesage et al., 1996; Lopes et al., 2001) and then it was corroborated by crystallographic structures obtained from K2P members (Brohawn et al., 2012; Miller and Long, 2012) (see Figure 1F).

Another particular feature of $\mathrm{K} 2 \mathrm{P}$ channels is the so-called cap structure that is formed by the extracellular M1-P1 linkers of each K2P monomer (Brohawn et al., 2012; Miller and Long, 2012). The elucidation of the crystal structure of TWIK-1 and TRAAK channels showed that the cap forms two tunnel-like side portals which serve as extracellular ion pathway (Brohawn et al., 2012; Miller and Long, 2012; Figure 1D).

Two structures recently solved at very high resolution confirmed the presence of the cap structure in TRAAK and TREK-2 channels (Brohawn et al., 2013; Dong et al., 2015). Nevertheless, a closer look at the cap structure revealed that the disposition of the helices (two per monomer, 
denoted as inner and outer helices, respectively) forming the cap structure in TRAAK and TREK-2 differs from that originally reported for TWIK-1 and TRAAK channels (Brohawn et al., 2013; Dong et al., 2015). The structural difference is based on whether the outer helix from each M1-P1 monomer interacts with the inner helix from the same (conventional configuration, Figure 1D) or the other monomer (swapped configuration, Figure 1E).

Little is known about the functional role and the molecular determinants responsible for the maintenance of the cap structure. The first study assessing in this matter by Lessage et al. showed that the M1-P1 linker form a particular coiledcoiled domain in TWIK-1 channels, where the two cysteine residues in position 69 from each monomer form an inter-chain disulfide bridge (Lesage et al., 1996). Moreover, the mutation of the cysteine residue in position 69 (C69) prevented the formation of covalent dimers and resulted in a loss of function in TWIK1 channels, suggesting that the M1-P1 linker plays an essential role in the channel function (Lesage et al., 1996). This particular domain was called by Lessage and co-workers as SID for selfinteracting domain (Figure 1B). The SID domain is conserved in all K2P channels (Figure 1C). The presence of covalent dimers was then corroborated in native tissue for TWIK-1, TREK-1 and TRAAK channels (Cluzeaud et al., 1998; Maingret et al., 2000; Reyes et al., 2000). The covalent dimerization has been also corroborated for recombinant TASK- 2 and KCNK7 channels (Salinas et al., 1999; Lesage et al., 2001).

Further sequence analysis of K2P channels showed that the cysteine residue equivalent to the $\mathrm{C} 69$ is not conserved in other members of the family of K2P channels (subfamilies TASK and THIK) (see Figure 1C). However, a recent work by Goldstein et al. provides evidence for the presence of a cap structure in $\mathrm{K} 2 \mathrm{P}$ channels that do not have a cysteine residue in its M1-P1 linker (Goldstein et al., 2016). Additionally, the mutation of the homologous cysteine to serine in the M1-P1 linker neither affected TASK-2 function (Niemeyer et al., 2003) nor the dimer formation of TWIK-2 and TREK-1 channels (Patel et al., 2000; Hwang et al., 2014). Together, the evidence exposed above suggest that the cysteine residue located in M1-P1 linker is not relevant for the formation and/or maintenance of the cap structure or the cap structure does not play a critical role in the function of K2P channels. However, the presence of the C69 in the SID domain is necessary to the covalent binding of interacting $\mathrm{K} 2 \mathrm{P}$ subunits and potentially increases the stability of the dimers. More structural data from the mutants described above would provide clues to the understanding of the importance of the cap structure to the channel function. The article published by Goldstein et al. studied by alanine-scanning mutagenesis the effect of mutations in the M1-P1 linker on TASK-1 activity (Goldstein et al., 2016). The data obtained in this screening was then used to build a model of the TASK-1 cap that showed a strong structural similarity to that reported by $\mathrm{x}$-crystallography for channels displaying a cysteine residue in the M1-P1 loop (Goldstein et al., 2016). Interestingly, the model showed that the M1-P1 linkers from each monomer form a coiled coil domain. Coiled-coils consist of two to five amphipathic $\alpha$-helices that twist around one another to form a supercoil (Burkhard et al., 2001). In the case of K2P channels, two $\alpha$-helices form a parallel left-handed coiled coil that is characterized by a sevenresidue periodicity (heptad repeat), with the occurrence of apolar residues preferentially in the first (a) and fourth (d) positions of the "heptad" (Burkhard et al., 2001). Consistent with such a particular domain in TASK-1, a severe loss of function was observed in TASK-1 upon mutation of residues L48, Y52, L54, S55, Y59, and L62 which are from "a" and "d" sites that are critical for the coiled-coil formation (Goldstein et al., 2016).

Little is known about the role of the cap structure in $\mathrm{K} 2 \mathrm{P}$ channels but it has been shown in many coiled coildisplaying proteins that this domain plays an essential role in oligomerization (Burkhard et al., 2001). Hence, it is plausible that the cap structure may play a critical role in K2P dimerization. On the other hand, there is also evidence for the importance of the cap structure in K2P channel activity (Morton et al., 2005; Gonzalez et al., 2013) and the insensitivity of K2P channels to classical $\mathrm{K}^{+}$blockers (e.g., TEA, 4AP or toxin), precluding the blockers access to the pore (Brohawn et al., 2012; Miller and Long, 2012).

Another equally interesting feature is that the K2P channels are able to assemble heterodimers in different tissues, where they account for a significant part of the native leak current. For example, TASK-1/TASK-3 heterodimers play an important role in chemoreception, acting as primary sensors of hypoxia and metabolic acidosis (Buckler et al., 2000; Czirjak and Enyedi, 2002; Berg et al., 2004). TWIK-1 may also interact with TASK1 and TASK-3 subunits in the cerebellum (Plant et al., 2012). TWIK-1/TREK-1 heterodimers generate passive conductance and cannabinoid-induced glutamate release in astrocytes (Hwang et al., 2014). The THIK-2/THIK-1 heterodimers contribute to cell excitability, rescuing the silent THIK-2 subunit (Blin et al., 2014). And recent studies have shown the heterodimeric assembly of members from the TREK subfamily (Blin et al., 2016; Levitz et al., 2016).

These heterodimers configurations present properties different from those observed in their corresponding homodimers, increasing the $\mathrm{K}^{+}$channel diversity. Thus, the K2P subunits of the same or distant subfamilies that show an overlapping tissue distribution might form heterodimers and produces active channels. Perhaps the new cap structures present in these heterodimeric configurations could affect the gating mechanism and, at least in part, explain the new properties.

In summary, the cap structure in K2P channels seem to be conserved but the function of the cap structure and the mechanism involved in its formation and maintenance might differ among the K2P members. The elucidation of other cap structures of other K2P channels might get clues into the functional implications of conventional vs. swapped cap structures. It is tempting to speculate that the configuration of the cap structure might confer unique features in K2P channels that have the ability to form functional heterodimeric complexes such as those formed by TWIK1/TASK-1/TASK-3 (Buckler et al., 2000; Czirjak and Enyedi, 2002; Berg et al., 2004; Plant et al., 2012), TWIK-1/TREK-1 (Hwang et al., 2014), THIK-2/THIK-1 (Blin et al., 2014) or TRAAK/TREK1/TREK2 (Blin et al., 2016; Levitz et al., 2016). 
A

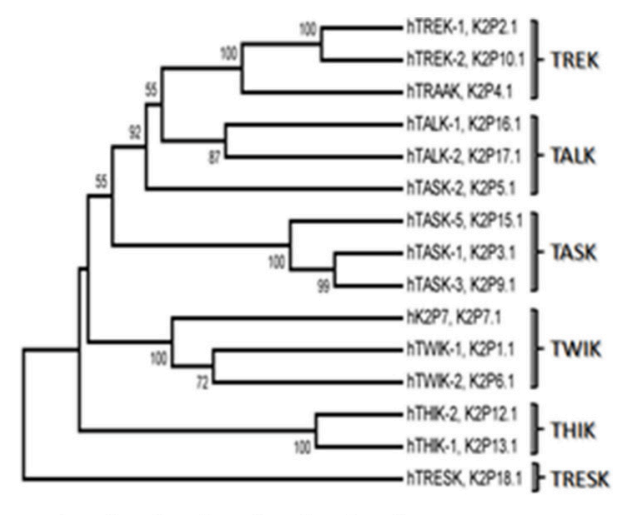

B

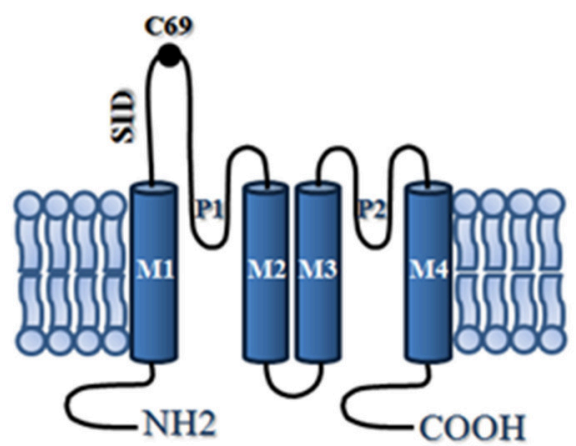

C
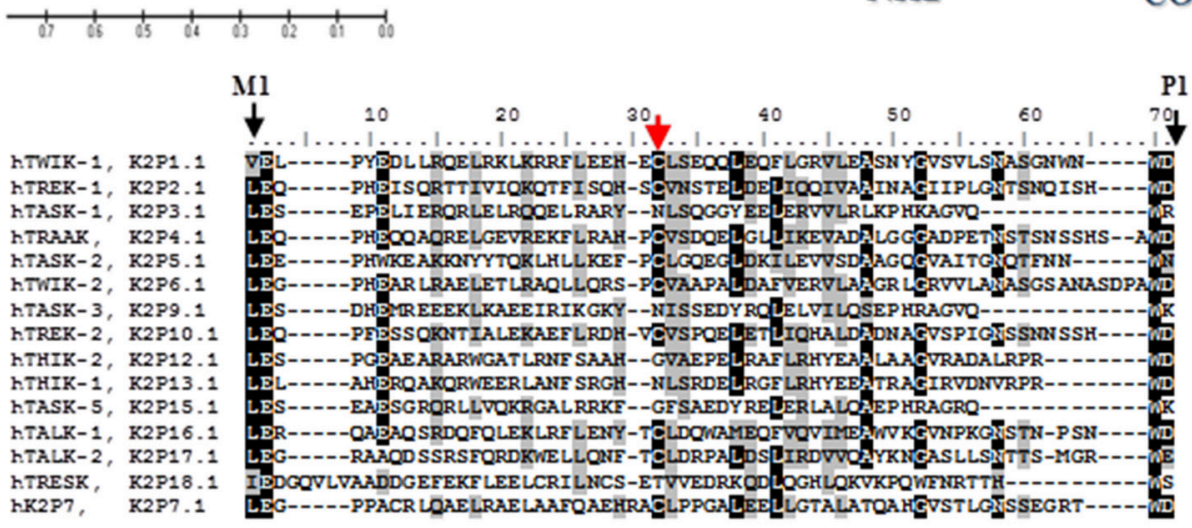

P1

KTWIK-1, K2P1

KTREK-1, K2P2

hTASK-1, K2P3.

KTRAAK, K2P4.

KTWTK-2, K2P6

HTASK $-3, \mathrm{~K} 2 \mathrm{Pg}$

LTREK- $2, \mathrm{~K} 2 \mathrm{P} 10$

मTRESK , K2P18

KK2P7, K2P7 ,
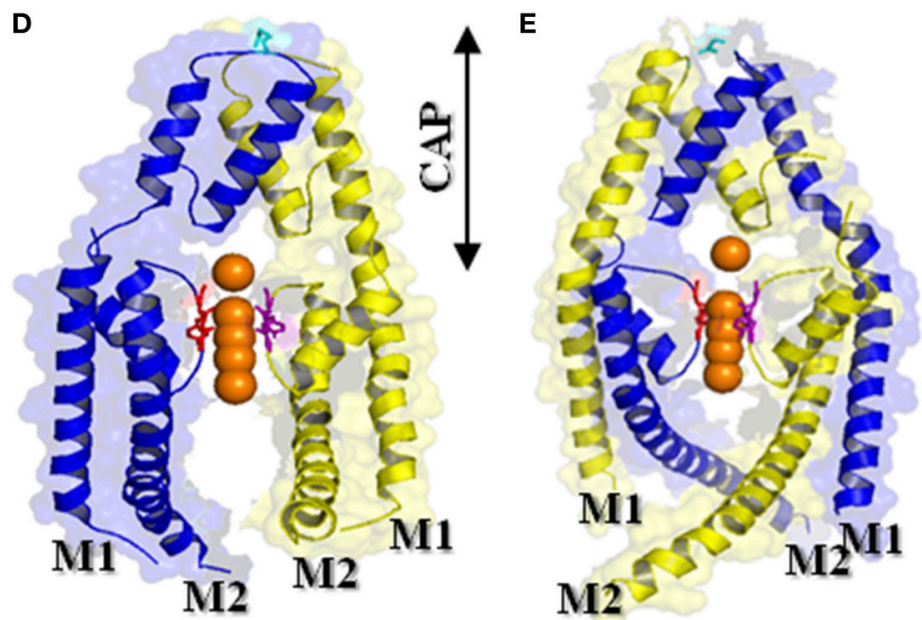

$\mathbf{F}$

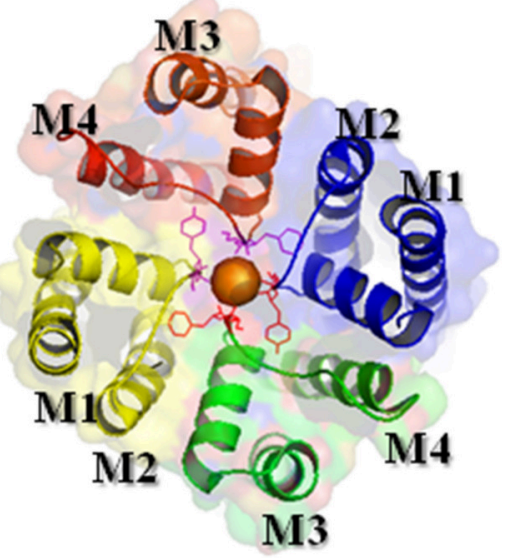

FIGURE 1 | K2P channels: (A) Phylogenetic tree for K2P family (the phylogenic analysis was done with MEGA software version 5). The length of lines indicates the relative distances between nodes and it is scaled below the tree. Numbers on branches indicate bootstrap values (as a percentage). (B) Topological model proposed for K2P channels, each subunit has two pore forming domain (P loops) and four transmembrane domains (denoted M1-M4). (C) Amino acid sequence alignment of K2P channel M1-P1 domain. Gaps are indicated by dashes, letters with black background are identical amino acids, and letters with gray background are similar amino acids. Red arrow indicates the cysteine residues involved in the formation of covalent bridge between two subunits. Amino acid identity (ID) among the M1-P1 domains of hTWIK-1 and the others K2P subunits was calculated. (D) View of the TRAAK structure (PDB code 3UM7) in ribbon representation, showing a non-swapped configuration (conventional configuration). (E) View of the TRAAK structure (PDB code 4I9W) in a domain swapped configuration. The potassium ions are colored orange and the chains are in blue and yellow. The disulfide bridge at the top of the cap is shown in stick representation colored cyan. (F) View from the extracellular side showing the TRAAK pore (PDB code 3UM7). The GYG sequence of each P domain is highlighted in stick representation and the helical cap was removed. For clarity in $(\mathbf{D}, \mathbf{E})$, the second pore domain was removed from each TRAAK subunits. 


\section{AUTHOR CONTRIBUTIONS}

All authors listed, have made substantial, direct and intellectual contribution to the work, and approved it for publication.

\section{REFERENCES}

Barel, O., Shalev, S. A., Ofir, R., Cohen, A., Zlotogora, J., Shorer, Z., et al. (2008). Maternally inherited Birk Barel mental retardation dysmorphism syndrome caused by a mutation in the genomically imprinted potassium channel KCNK9. Am. J. Hum. Genet. 83, 193-199. doi: 10.1016/j.ajhg.2008.0 7.010

Berg, A. P., Talley, E. M., Manger, J. P., and Bayliss, D. A. (2004). Motoneurons express heteromeric TWIK-related acid-sensitive $\mathrm{K}+$ (TASK) channels containing TASK-1 (KCNK3) and TASK-3 (KCNK9) subunits. J. Neurosci. 24, 6693-6702. doi: 10.1523/JNEUROSCI.1408-04.2004

Blin, S., Ben, S., Kim, E. J., Brau, F., Kang, D., Bichet, D., et al. (2016). Mixing and matching TREK/TRAAK subunits generate heterodimeric K2P channels with unique properties. Proc. Natl. Acad. Sci. U.S.A 113, 4200-4205. doi: $10.1073 /$ pnas. 1522748113

Blin, S., Chatelain, F. C., Feliciangeli, S., Kang, D., Lesage, F., and Bichet, D. (2014). Tandem pore domain halothane-inhibited $\mathrm{K}+$ channel subunits THIK1 and THIK2 assemble and form active channels. J. Biol. Chem. 289, 28202-28212. doi: 10.1074/jbc.M114.600437

Brohawn, S. G., Campbell, E. B., and MacKinnon, R. (2013). Domain-swapped chain connectivity and gated membrane access in a Fab-mediated crystal of the human TRAAK K+ channel. Proc. Natl. Acad. Sci. U.S.A 110, 2129-2134. doi: $10.1073 /$ pnas. 1218950110

Brohawn, S. G., del Mármol, J., and MacKinnon, R. (2012). Crystal structure of the human K2P TRAAK, a lipid- and mechano-sensitive $\mathrm{K}+$ ion channel. Science 335, 436-441. doi: 10.1126/science. 1213808

Buckler, K. J., Williams, B. A., and Honore, E. (2000). An oxygen-, acidand anaesthetic-sensitive TASK-like background potassium channel in rat arterial chemoreceptor cells. J. Physiol. 525(Pt 1), 135-142. doi: 10.1111/j.14697793.2000.00135.x

Burkhard, P., Stetefeld, J., and Strelkov, S. V. (2001). Coiled coils: a highly versatile protein folding motif. Trends Cell Biol. 11, 82-88. doi: 10.1016/S09628924(00)01898-5

Cluzeaud, F., Reyes, R., Escoubet, B., Fay, M., Lazdunski, M., Bonvalet, J. P., et al. (1998). Expression of TWIK-1, a novel weakly inward rectifying potassium channel in rat kidney. Am. J. Physiol. 275, C 1602-C1609.

Czirják, G., and Enyedi, P. (2002). Formation of functional heterodimers between the TASK-1 and TASK-3 two-pore domain potassium channel subunits. J. Biol. Chem. 277, 5426-5432. doi: 10.1074/jbc.M107138200

Dong, Y. Y., Pike, A. C., Mackenzie, A., McClenaghan, C., Aryal, P., Dong, L., et al. (2015). K2P channel gating mechanisms revealed by structures of TREK2 and a complex with Prozac. Science 347, 1256-1259. doi: 10.1126/science.12 61512

Enyedi, P., and Czirják, G. (2010). Molecular background of leak K+ currents: two-pore domain potassium channels. Physiol. Rev. 90, 559-605. doi: 10.1152/physrev.00029.2009

Feliciangeli, S., Chatelain, F. C., Bichet, D., and Lesage, F. (2015). The family of K2P channels: salient structural and functional properties. J. Physiol. 593, 2587-2603. doi: 10.1113/jphysiol.2014.287268

Goldstein, M., Rinné, S., Kiper, A. K., Ramírez, D., Netter, M. F., Bustos, D., et al. (2016). Functional mutagenesis screens reveal the 'cap structure' formation in disulfide-bridge free TASK channels. Sci. Rep. 6, 19492. doi: 10.1038/srep 19492

Goldstein, S. A., Bayliss, D. A., Kim, D., Lesage, F., Plant, L. D., and Rajan, S. (2005). International union of pharmacology. LV. Nomenclature and molecular relationships of two-P potassium channels. Pharmacol. Rev. 57, 527-540. doi: $10.1124 /$ pr.57.4.12

Goldstein, S. A., Bockenhauer, D., O'Kelly, I., and Zilberberg, N. (2001). Potassium leak channels and the KCNK family of two-P-domain subunits. Nat. Rev. Neurosci. 2, 175-184. doi: 10.1038/35058574

\section{ACKNOWLEDGMENTS}

We thank Dr. Marcelo A. Catalán for critical reading of the manuscript, helpful comments and stimulating discussions.

Gonzalez, W., Zuniga, L., Cid, L. P., Arevalo, B., Niemeyer, M. I., and Sepulveda, F. V. (2013). An extracellular ion pathway plays a central role in the cooperative gating of a K2P K+ channel by extracellular pH. J. Biol. Chem. 288, 5984-5991. doi: 10.1074/jbc.M112.445528

Hwang, E. M., Kim, E., Yarishkin, O., Woo, D. H., Han, K. S., Park, N., et al. (2014). A disulphide-linked heterodimer of TWIK-1 and TREK-1 mediates passive conductance in astrocytes. Nat. Commun. 5, 3227. doi: 10.1038/ncomms4227

Lafrenière, R. G., Cader, M. Z., Poulin, J. F., Andres-Enguix, I., Simoneau, M., Gupta, N., et al. (2010). A dominant-negative mutation in the TRESK potassium channel is linked to familial migraine with aura. Nat. Med. 16, 1157-1160. doi: 10.1038/nm.2216

Lesage, F., and Lazdunski, M. (2000). Molecular and functional properties of twopore-domain potassium channels. Am. J. Physiol. Renal Physiol. 279, F793F801.

Lesage, F., Reyes, R., Fink, M., Duprat, F., Guillemare, E., and Lazdunski, M. (1996). Dimerization of TWIK-1 K+ channel subunits via a disulfide bridge. EMBO J. 15, 6400-6407.

Lesage, F., Reyes, R., Lazdunski, M., and Barhanin, J. (2001). Leak K+ channels with two pore domains. Kidney Blood Press. Res. 24, 402.

Levitz, J., Royal, P., Comoglio, Y., Wdziekonski, B., Schaub, S., Clemens, D. M., et al. (2016). Heterodimerization within the TREK channel subfamily produces a diverse family of highly regulated potassium channels. Proc. Natl. Acad. Sci. U.S.A. 113, 4194-4199. doi: 10.1073/pnas.15224 59113

Lopes, C. M., Zilberberg, N., and Goldstein, S. A. (2001). Block of Kcnk3 by protons. Evidence that 2-P-domain potassium channel subunits function as homodimers. J. Biol. Chem. 276, 24449-24452. doi: 10.1074/jbc.C1001 84200

Lotshaw, D. P. (2007). Biophysical, pharmacological, and functional characteristics of cloned and native mammalian two-pore domain $\mathrm{K}+$ channels. Cell Biochem. Biophys. 47, 209-256. doi: 10.1007/s12013-007-0007-8

Ma, L., Roman-Campos, D., Austin, E. D., Eyries, M., Sampson, K. S. Soubrier, F., et al. (2013). A novel channelopathy in pulmonary arterial hypertension. N. Engl. J. Med. 369, 351-361. doi: 10.1056/NEJMoa12 11097

Maingret, F., Lauritzen, I., Patel, A. J., Heurteaux, C., Reyes, R., Lesage, F., et al. (2000). TREK-1 is a heat-activated background $\mathrm{K}(+)$ channel. EMBO J. 19, 2483-2491. doi: 10.1093/emboj/19.11.2483

Miller, A. N., and Long, S. B. (2012). Crystal structure of the human two-pore domain potassium channel K2P1. Science 335, 432-436. doi: $10.1126 /$ science. 1213274

Morton, M. J., Abohamed, A., Sivaprasadarao, A., and Hunter, M. (2005). pH sensing in the two-pore domain K+ channel, TASK2. Proc. Natl. Acad. Sci. U.S.A. 102, 16102-16106. doi: 10.1073/pnas.0506870102

Mu, D., Chen, L., Zhang, X., See, L. H., Koch, C. M., Yen, C., et al. (2003). Genomic amplification and oncogenic properties of the KCNK9 potassium channel gene. Cancer Cell 3, 297-302. doi: 10.1016/S1535-6108(03)00054-0

Niemeyer, M. I., Cid, L. P., Valenzuela, X., Paeile, V., and Sepúlveda, F. V. (2003). Extracellular conserved cysteine forms an intersubunit disulphide bridge in the KCNK5 (TASK-2) K+ channel without having an essential effect upon activity. Mol. Membr. Biol. 20, 185-191. doi: 10.1080/09687680310000 84181

Patel, A. J., Maingret, F., Magnone, V., Fosset, M., Lazdunski, M., and Honoré, E. (2000). TWIK-2, an inactivating $2 \mathrm{P}$ domain K+ channel. J. Biol. Chem. 275, 28722-28730. doi: 10.1074/jbc.M003755200

Plant, L. D., Zuniga, L., Araki, D., Marks, J. D., and Goldstein, S. A. (2012). SUMOylation silences heterodimeric TASK potassium channels containing K2P1 subunits in cerebellar granule neurons. Sci. Signal. 5, ra84. doi: 10.1126/scisignal.2003431

Reyes, R., Lauritzen, I., Lesage, F., Ettaiche, M., Fosset, M., and Lazdunski, M. (2000). Immunolocalization of the arachidonic acid and mechanosensitive 
baseline traak potassium channel in the nervous system. Neuroscience 95, 893-901. doi: 10.1016/S0306-4522(99)00484-4

Salinas, M., Reyes, R., Lesage, F., Fosset, M., Heurteaux, C., Romey, G., et al. (1999). Cloning of a new mouse two-P domain channel subunit and a human homologue with a unique pore structure. J. Biol. Chem. 274, 11751-11760. doi: 10.1074/jbc.274.17.11751

Schmidt, C., Wiedmann, F., Voigt, N., Zhou, X. B., Heijman, J., Lang, S., et al. (2015). Upregulation of $\mathrm{K}(2 \mathrm{P}) 3.1 \mathrm{~K}+$ current causes action potential shortening in patients with chronic atrial fibrillation. Circulation 132, 82-92. doi: 10.1161/CIRCULATIONAHA.114.012657
Conflict of Interest Statement: The authors declare that the research was conducted in the absence of any commercial or financial relationships that could be construed as a potential conflict of interest.

Copyright (๑ 2016 Zúñiga and Zúñiga. This is an open-access article distributed under the terms of the Creative Commons Attribution License (CC BY). The use, distribution or reproduction in other forums is permitted, provided the original author(s) or licensor are credited and that the original publication in this journal is cited, in accordance with accepted academic practice. No use, distribution or reproduction is permitted which does not comply with these terms. 